\title{
Söders Ökofeuerwerk und die Grünfärbung der CSU: Diskursnetzwerke im bayrischen Themenwettbewerb
}

\author{
Gina-Julia Westenberger $(\mathbb{D} \cdot$ Volker Schneider
}

Eingegangen: 18. Januar 2021 / Überarbeitet: 13. Dezember 2021 / Angenommen: 17. Dezember 2021 /

Online publiziert: 13. Januar 2022

(C) Der/die Autor(en) 2022

Zusammenfassung Umwelt- und Klimapolitik ist in den letzten Jahren zu einem äußerst relevanten Themenfeld des Parteienwettbewerbs in Deutschland avanciert, an welchem sich gut beobachten lässt, welche bedeutende Rolle spezifische Themen und Probleme im Wettbewerb um Wählerstimmen spielen. In diesem Artikel demonstrieren wir erstmalig, wie die Methode der Diskursnetzwerkanalyse zur Analyse solcher Themenwettbewerbe eingesetzt werden kann. Diskursnetzwerkanalysen verbinden die qualitative Inhaltsanalyse von Medienberichten mit Methoden der Sozialen Netzwerkanalyse und erlauben es so, über exakte Zeitfenster hinweg die Dynamik eines Themenwettbewerbs und die Interaktion von Parteien detailliert zu verfolgen und zu vergleichen. Dieses Potenzial demonstrieren wir am Beispiel des Issue-Wettbewerbs in der bayrischen Umweltpolitik in den Jahren 2018 und 2019. Ein besonderer Fokus liegt dabei auf der staatstragenden CSU, die im Landtagswahlkampf 2018 noch versuchte, mit ausgeprägten migrationspolitischen Forderungen Wähler der AfD abzuwerben. Ein Jahr später zündete Ministerpräsident und Parteichef Markus Söder hingegen ein ganzes „Feuerwerk“ an umwelt- und klimapolitischen Vorschlägen. Wie kam es zu dieser Neuausrichtung des Diskurses? Anhand von Zeitungsartikeln aus der Süddeutschen Zeitung und im Vergleich dreier Diskursperioden zeigen wir, wie sich die Salienz der Umweltpolitik im Diskursverlauf veränderte. Eine entscheidende Rolle spielte dabei vor allem das überaus erfolgreiche Volksbegehren „Rettet die Bienen“, infolgedessen sich der umweltpolitische Diskurs deutlich intensivierte und diversifizierte. Unsere Analyse zeigt, dass sich die CSU bedingt durch diese Dynamik und die Bedrohung durch die elektoralen Erfolge der Grünen, daraufhin gezwungen sah mit diesen in einen Wettkampf um die Issue-Ownership grüner Themen einzutreten.

Gina-Julia Westenberger $(\bowtie) \cdot$ Volker Schneider Fachbereich Politik- und Verwaltungswissenschaft, Universität Konstanz,

Universitätsstraße 10, 78464 Konstanz, Deutschland

E-Mail: gina-julia.westenberger@uni-konstanz.de 
Schlüsselwörter CSU · Bayern · Diskursnetzwerkanalyse · Issue-Competition · Issue-Ownership · Umweltpolitik

\title{
Söder's ecological fireworks and the greenification of the CSU: discourse networks in the bavarian issue competition
}

\begin{abstract}
Environmental and climate policy has become an important issue in party competition in Germany in recent years, and it is easy to observe the significant role that specific issues and problems play in the competition for votes within this policy area. In this article, we demonstrate for the first time how the method of discourse network analysis can be used to analyse such issue competitions. Discourse network analysis combines qualitative content analysis of media reports with methods of social network analysis and thus allows to follow and compare the dynamics of an issue competition and the interaction of parties in detail over exact time windows. We demonstrate this potential using the example of issue competition in Bavarian environmental politics in 2018 and 2019. We place a particular focus on the mainstream party CSU, which in the 2018 state election campaign was still trying to lure away voters from the AfD with pronounced migration policy demands. A year later, on the contrary, Prime Minister and party leader Markus Söder, ignited a whole "firework" of environmental and climate policy proposals. How did this reorientation of the discourse come about? Using newspaper articles from the Süddeutsche Zeitung and comparing three discourse periods, we show how the salience of environmental policy changed over the course of the discourse. The extremely successful petition for a referendum "Save the Bees" played a decisive role in this process, as a result of which the environmental policy discourse intensified and diversified significantly. Our analysis shows that because of this dynamic, and the threat posed by the electoral successes of the Greens, the CSU felt compelled to enter into a competition with the Greens for the issue ownership of green issues.
\end{abstract}

Keywords CSU · Bavaria · Discourse network analysis · Issue competition · Issue ownership · Environmental policy

\section{Einleitung}

Seit seinem Aufstieg auf die politische Tagesordnung ist Umwelt- und Klimapolitik zu einem wichtigen Themenfeld des Parteienwettbewerbs avanciert. Nicht nur die Grünen, sondern auch die übrigen deutschen Parteien konkurrieren, wenn auch in unterschiedlicher Intensität, in der Wählerschaft um Kompetenzzuschreibungen in diesem Politikbereich. Einen sehr interessanten Fall des Themenwettbewerbs stellt die ökologische Wende der CSU im Frühling 2019 dar. Weniger als ein Jahr zuvor hatte die Partei sich noch mit Parolen wie „Asyltourismus“ und dem Aufhängen von Kruzifixen in Landesbehörden in Richtung der Wähler des rechteren Parteienspektrums orientiert. Nach dem überaus erfolgreichen Volksbegehren „Rettet die Bienen“ im Februar 2019 zündete der bayrische Ministerpräsident Markus Söder mit Forderungen nach einem vorgezogenen Kohleausstieg bis 2030, mehrwertsteuerbefreiten 
Bahntickets oder der Pflanzung von 30Mio. Bäumen in Bayern jedoch ein wahres „grünes Feuerwerk“ um im Wettbewerb der bayerischen Parteien ein neues Thema zu besetzen (Bauchmüller 2019; Neff 2018).

Dieser Entwicklung gehen wir nach und untersuchen auf der Ebene des politischen Mediendiskurses, wie es in einem Vergleich von drei Diskursphasen zu dieser thematischen Neuausrichtung kam. Für die Erklärung greifen wir auf die Theorien der Issue-Ownership und der Issue-Competition zurück und tracen den Positionsund Themenwechsel mit der Methode der Diskursnetzwerkanalyse, die qualitative Inhaltsanalyse von Medienberichten mit Methoden der Sozialen Netzwerkanalyse verbindet. Damit bieten wir die erste Diskursnetzwerkanalyse, die aus der Themenwettbewerbsperspektive durchgeführt wird, und verfolgen damit einen neue Variante der Analyse von "Issue-Networks“, die bereits in den 1970er-Jahren die Politiknetzwerkforschung stimulierte (Schneider 2015; Heclo 1978).

Wir gehen dabei wie folgt vor: Zunächst skizzieren wir den umweltpolitischen Kontext des Diskurses in Bayern. Anschließend erläutern wir unseren theoretischen Rahmen, der auf der Forschung zur Issue-Ownership und Issue-Competition beruht, und leiten daraus vier Hypothesen zum Diskursverhalten der Parteien ab. Darauffolgend stellen wir in Kürze die Methode der Diskursnetzwerkanalyse sowie das Vorgehen in der inhaltsanalytischen Datengenerierung vor. Wir werden sodann die Positionen der Parteien in Bezug auf Konzepte und Themen in den Diskursphasen vergleichen, und die thematischen Wettbewerbsbeziehung der bayerischen Parteien netzwerkanalytisch herausarbeiten. In den letzten beiden Abschnitten werden die wichtigsten Ergebnisse sowie der Beitrag und die Limitationen unserer Analyse diskutiert.

\section{Umweltpolitik und Parteienwettbewerb in Bayern}

\subsection{Historischer Abriss}

In unserem Beitrag geht es um die Positionierung der CSU und ihrer parteipolitischen Konkurrenten im Themenfeld der Umweltpolitik, zu dem zahlreiche subdomains wie Klimapolitik, Natur- und Artenschutz bis hin zur Kontrolle von Umweltchemikalien gehören. Hierzu ist es sinnvoll, zuerst den historischen Hintergrund der Umweltpolitik im bayerischen Parteienwettbewerb zu betrachten.

Bayern nahm lange Zeit eine Vorreiterrolle im Umweltschutz ein. Bereits 1946 enthielt die bayrische Landesverfassung folgenden Bestimmung zum Naturschutz: „Der deutsche Wald, kennzeichnende Orts- und Landschaftsbilder und die einheimischen Tier- und Pflanzenarten sind möglichst zu schonen und zu erhalten" (Art. 141 Abs. 2). Wurde dieser Passus noch auf Drängen des späteren bayrischen Ministerpräsidenten Wilhelm Hoegner von der SPD eingebracht (Egleder 2010), so ist für die spätere Entwicklung des Umweltschutzes vor allem die lange Regierungszeit der CSU entscheidend, die seit 1957 durchgehend die Regierung stellt.

Die Gründung des weltweit ersten eigenen „Staatsministeriums für Landesentwicklung und Umweltfragen“ im Jahre 1970 erfolgte primär mit der Zielsetzung, die wirtschaftliche Entwicklung in Niederbayern mit der Schonung der dortigen 
Natur in Einklang zu bringen. Der Fokus des Ministeriums lag deutlich auf der Landesentwicklung, aber nichtdestotrotz wurde im selben Jahr der erste Nationalpark Deutschlands im Bayrischen Wald gegründet (Egleder 2010). Wenig später gab sich das Land mit dem bayrischen Naturschutzgesetz von 1973 die damals europaweit modernste Gesetzgebung zum Naturschutz; der Bund folgte erst drei Jahre später mit einem eigenen Naturschutzgesetz (Glück 2006). Im selben Jahrzehnt legte Bayern ein eigenes Umweltprogramm vor (Bayrisches Staatsministerium für Umwelt und Verbraucherschutz 2020). Seit dieser Zeit wird ,die ,Verantwortung für die Schöpfung ‘ als Motivation und der ,Schutz der natürlichen Lebensgrundlagen“ als Politikziel“" in jedem Parteiprogramm der CSU angeführt (Egleder 2010, S. 207). Obwohl mit einer christlichen Rhetorik versehen, lässt sich der verstärkte Umweltschutz-Fokus der CSU als Reaktion auf die zunehmende Relevanz des Themas in der Bevölkerung und vor allem auf die Gründung der Grünen im Jahr 1980 werten. Angeregt durch die kontroversen Diskussionen zum „Waldsterben“ und zum „Sauren Regen“ der 1980er-Jahre nahm Bayern 1984 als erstes Bundesland den „Schutz der natürlichen Lebensgrundlagen“ als Staatsziel in seine Verfassung auf (Glück 2006). Bereits ein Jahr zuvor war der Bayrische Naturschutzfonds eingerichtet worden. Im Jahr 2008 wurde eine spezielle Biodiversitätsstrategie beschlossen, welche durch das Programm „NaturVielfaltBayern“ aus dem Jahr 2014 weiter umgesetzt wird (Bayrisches Staatsministerium für Umwelt und Verbraucherschutz 2020).

Trotz dieser Anstrengungen für den Umweltschutz fällt deren Bewertung gemischt aus. Kohout (2013) kritisiert, dass der Umweltschutz zu oft zugunsten von Wirtschafts- und Agrarinteressen vernachlässigt werde. Nach Egleder (2010, S. 213) findet Umweltschutz in Bayern hauptsächlich ,dort statt, wo es nicht weh tut“" und sie vermutet, dass hinter der „Verantwortung für die Schöpfung“ (S. 207) im CSUProgramm hauptsächlich das strategische Interesse steckt, im Themenwettbewerb mit den Grünen verstärkt grüne Positionen einzunehmen.

\subsection{Stand des bayrischen Parteienwettbewerbs 2018-2019}

Im Sommer 2018, dem Beginn unseres Untersuchungszeitraums, spielten umweltpolitische Themen noch eine Nebenrolle in der politischen Kommunikation der CSU. Stattdessen konzentrierte sich Markus Söder durch seine Beteiligung am Asylstreit der Union auf das Themenfeld Migration. So plädierte er insbesondere dafür, ,Asyltourismus“ zu bekämpfen. Mit diesem Kampfbegriff rechter Parteien versuchte die CSU, in der Landtagswahl im Herbst AfD-Wähler für die Partei zurückzugewinnen.

Auch wenn nach den Ausschreitungen in Chemnitz im August 2018 die Wahltaktik verändert, und jegliche Form der Zusammenarbeit mit der AfD ausgeschlossen wurde, scheiterte diese Strategie: Bei den Wahlen am 14. Oktober 2018 verlor die CSU fast zehn Prozent ihrer Stimmen und erreichte mit 37,2\% ihr schlechtestes Ergebnis seit 1950. Die AfD hingegen schaffte mit 10,2\% erstmals den Sprung in den Landtag (Bayrischer Landtag 2018). Durch die Verluste der SPD von fast $11 \%$ und ihrem eigenen Plus von neun Prozent wurden zudem die Grünen mit 17,6\% zum ersten Mal zweistärkste Kraft im Bayrischen Landtag. Zudem konnte die FDP mit 5,1\% ihren Wiedereinzug in den Landtag feiern. 
Neben anderen Einflüssen, wie Popularitätsverlusten von Horst Seehofer und der unionsgeführten Bundesregierung, kann die Relevanz des Themenbereiches Umwelt- und Klimapolitik als ein bestimmender Faktor dieses Wahlausganges betrachtet werden. So gaben $49 \%$ der bayrischen Wähler die Themen Umwelt- und Klimapolitik als wichtiges Thema bei der Landtagswahl 2018 an, nur 33\% betrachteten hingegen die Flüchtlings- und Asylpolitik als ausschlaggebend für ihre Wahlentscheidung. Dieser Trend war von der CSU schlichtweg verkannt worden. Zwar hatten vor der Landtagswahl alle Parteien das Thema Naturschutz in ihr Wahlprogramm aufgenommen, eine intensive Beschäftigung erfuhr das Thema allerdings nur durch die Grünen, sowie die SPD, Linke und die Ökologisch-Demokratische Partei (ÖDP). Die infolgedessen erstmals in Koalition mit den ebenfalls erstarkten Freien Wählern (FW) gebildete Regierung von Ministerpräsident Söder nahm am 12. November 2018 ihre Amtsgeschäfte auf.

Nur wenige Monate später drängte sich das Umweltschutzthema wieder in den Vordergrund. Nachdem das von der ÖDP initiierte Volksbegehren „Artenschutz und Naturschönheit in Bayern - Rettet die Bienen“ Mitte November zur Abstimmung zugelassen worden war, bestand vom 31. Januar bis zum 13. Februar 2019 die Möglichkeit, sich in den Rathäusern als Unterstützer eintragen zu lassen. Ziel des Volksbegehrens war eine Änderung des Bayrischen Naturschutzgesetzes zugunsten von mehr Artenschutz. Wesentliche Forderungen umfassten eine Steigerung der ökologischen Landwirtschaft (auf 30\% bis 2030), die Schaffung eines 13\% der Agrarfläche umfassenden Biotopverbundes, die Einführung eines fünf Meter breiten Schutzstreifens an Gewässern, und das Verbot von Pestiziden in Naturschutzgebieten. Insgesamt beteiligten sich 1,75 Mio. Bayern, womit fast jeder fünfte Wahlberechtigte teilnahm (Bayrisches Landesamt für Statistik 2019).

„Rettet die Bienen“wurde durch die breite Unterstützung von sowohl ländlichen als auch urbanen Bevölkerungsschichten zum erfolgreichsten Volksbegehren in der Geschichte Bayerns (Bayrisches Landesamt für Statistik 2019). Die Staatsregierung reagierte schnell: Bereits eine Woche nach dem Ende des Abstimmungszeitraumes berief Ministerpräsident Söder einen Runden Tisch ein, um mit den Initiatoren des Volksbegehrens, Vertretern des einflussreichen Bayrischen Bauernverbandes (BBV) und Regierungsmitgliedern Möglichkeiten zum „Schutz von Bienen und Bauern“ auszuloten. Eine Einigung wurde schnell erzielt, wodurch die Regierung einen Volksentscheid umging. Am 24. Juli 2019 verabschiedete der Landtag die „Änderung des Bayrischen Naturschutzgesetztes zugunsten der Artenvielfalt und Naturschönheit in Bayern“, mit welcher man sich etwa auch dem Ziel einer klimaneutralen Staatsverwaltung bis zum Jahr 2030 verpflichtete (Art. 11c BayNatSchG). Begleitet wurde die Annahme des Textes von einem „Versöhnungsgesetz“, welches einen Interessensausgleich mit den Landwirten zum Ziel hatte. So wurden etwa Ausnahmeregelung für die unter Landwirten sehr umstrittenen Wiesen-Walzverbote nach dem 15. März eingeführt (Art. 3 Abs. 6 BayNatSchG).

Neben der Landtagswahl und dem Volksbegehren fand mit der Wahl zum Europäischen Parlament am 26. Mai 2019 ein drittes elektorales Ereignis statt, das den umweltpolitischen Diskurs beeinflusste. Bei der Europawahl konnte die CSU mit einem zarten Plus von 0,2\% auf insgesamt 40,7\% der Stimmen den bisherigen Abwärtstrend aufhalten. Als großer Sieger der Wahlen zeigten sich erneut die Grünen, 


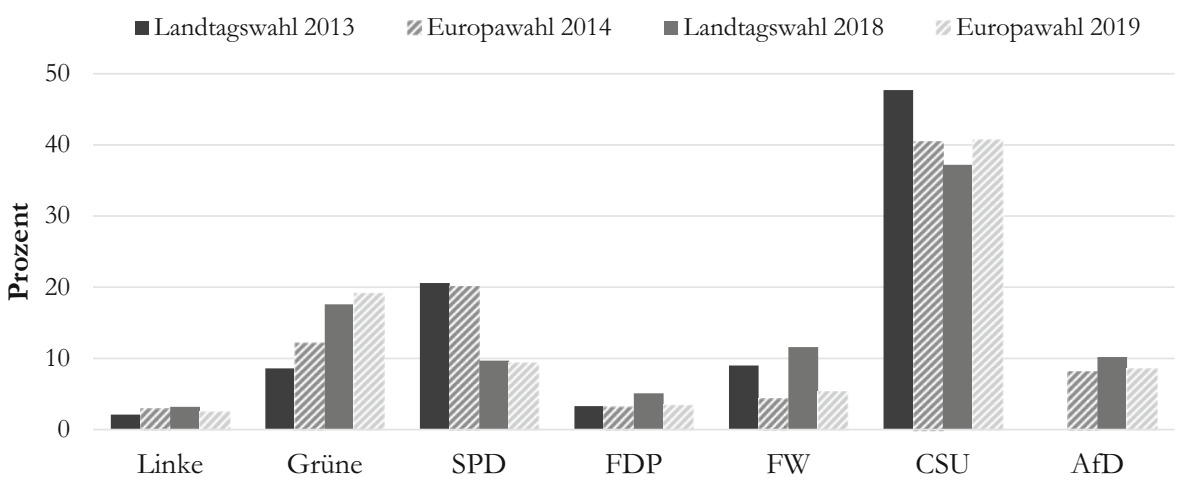

Abb. 1 Wahlergebnisse der größten bayrischen Parteien im Vergleich (Angaben in Prozent)

welche mit einem Ergebnis von 19,1\% wie bereits bei der Landtagswahl an zweite Stelle hinter die CSU aufschlossen. Der Stimmenanteil der SPD halbierte sich auf 9,3\%, AfD, FW, FDP, Linke und ÖDP verzeichneten weder deutliche Gewinne noch Verluste (Bundeswahlleiter 2019) (siehe auch Abb. 1).

\section{Parteienkonkurrenz durch Problemkompetenz und Themenwettbewerb}

Zwischen der Landtagswahl und der Europawahl hat die CSU eine Reihe von umweltpolitischen Positionen verändert. Eine unserer zentralen Annahmen ist, dass dies nicht nur durch Policy-Learning mittels Überzeugungswandel (Sabatier und Weible 2007) oder Politikdiffusion (Lütz 2007) aus anderen Ländern oder Regionen verursacht wurde, sondern vor allem dem Kalkül entsprach, im Themenwettbewerb umweltpolitische Issue-Ownership zu gewinnen. Unsere Analyse fokussiert daher auf den thematischen Strategien parteipolitischer Akteure im medialen Diskurs, spezifische Themen $\mathrm{zu}$ besetzen, zu verteidigen oder auch $\mathrm{zu}$ unterminieren, um sich Vorteile im elektoralen Stimmenmarkt zu sichern.

Die Dynamik des Parteienwettbewerbs ist heute komplexer als noch vor 50 Jahren. Die Gründe liegen in der zunehmenden Volatilität der Wähler, der schwindenden Parteibindung und der augenscheinlich abnehmenden ideologischen Distanz zwischen den Parteien (Lefevere et al. 2015). Wahlkämpfe werden heute nicht mehr nur durch feste gesellschaftliche Spaltungen strukturiert, sondern zunehmend auch durch wechselnde Problemfragen (Dalton 1996; Dalton und Wattenberg 2000; Green-Pedersen 2007). Die Prognose von Wahlverhalten wird daher erschwert. Issue-Ownership- und Issue-Competition-Ansätze mit ihrem Fokus auf Themenkompetenz können Erklärungsansätze liefern, die auch kurzfristige Veränderungen erfassen können.

Die Ursprünge der Issue-Ownership-Theorie lassen sich bis in die 1950er-Jahre zurückverfolgen. Grundlegende Theoreme dieses Ansatzes wurden im Wesentlichen von Robertson (1976) zusammengefasst. In der Analyse britischer Parteiprogramme stellte dieser fest, dass Parteien eine direkte thematische Konfrontation mit ihren politischen Gegnern im Wahlkampf vermieden, und stattdessen nur auf jene The- 
men Bezug nahmen, die ihrer politischen Agenda nützten. Budge und Farlie (1983) griffen diese Beobachtungen mit ihrer Salienz-Theorie auf, nach der politische Parteien ihre Politikinhalte durch spezifische Themen hervorheben und andere, für sie ungünstiger erscheinende Themen vermieden. Petrocik (1996) präzisierte diese Annahmen dergestalt, dass Wahlentscheidungen zunehmend vom Problembewusstsein der Wähler in Bezug auf bestimmte Themen beeinflusst würden. Dieses Problembewusstsein sei im Gegensatz zu ideologischen Policy-Orientierungen schneller wandelbar, und könne so auch durch spezifische Ansprache in politischen Kampagnen beeinflusst bzw. verstärkt werden (Petrocik 1996; Smith 1985). Ferner geht diese Theorie davon aus, dass eine Partei - gewollt oder ungewollt - mit den Themen assoziiert wird, bezüglich derer ihr die Wähler die größte Kompetenz zuschreiben. Parteien haben daher Besitz- oder Eigentumsbeziehungen zu Themen und konzentrieren sich im Wahlkampf entsprechend auf jene, mit denen sie positiv assoziiert werden. Andere Themen werden nicht erwähnt, um beim Wähler keine gedankliche Verbindung zu politischen Gegnern herzustellen, welche bei diesen Themen IssueOwnership-Vorteile besitzen.

Die meisten Arbeiten zu Issue-Ownership beleuchten allerdings nur das Verhalten einzelner Parteien (Riker 1996; Meguid 2005) und blenden deren Interaktionen miteinander oft aus (Green-Pedersen und Mortensen 2010). Um diese hervorzuheben, führten Green-Pedersen und Mortensen (2010) das Konzept der „ParteiensystemAgenda" ein und argumentieren, dass aus der kontinuierlichen politischen Debatte im politischen System eine Agenda an Themen erwächst, auf welche die Parteien in jedem Fall Bezug nehmen müssen. Medien und Wähler würden eine Antwort auf saliente Themen verlangen, das schlichte Ignorieren eines Themas würde daher einer politischen Unfähigkeitserklärung gleichkommen. Aber auch eine positive „Umdeutung“ eines Themas erfordere eine kommunikative Handlung. In ihrer Studie zum dänischen Themenwettbewerb kommen sie zum Schluss, dass die Themen auf der Agenda des Parteiensystems klar von der Opposition beherrscht würden. Oppositionsparteien hätten mehr Möglichkeiten, ihre Themen durch kontinuierliche Betonung auf die Agenda zu bringen und die Regierung zu kritisieren, während letztere häufig auf externe Entwicklungen reagieren müsste, und dadurch weniger Chancen hätte, ihre präferierten Themen zu betonen (Green-Pedersen und Mortensen 2010).

Andererseits haben Regierungsparteien viele Möglichkeiten, im Themenwettbewerb durch die Ankündigung von politischen Maßnahmen ihre Problemkompetenz zu erhöhen, oder durch bestimmte politische Entscheidungen die Bedeutung von Themen selbst maßgeblich zu beeinflussen - bis hin zu deren Beseitigung. Betrachtet man die Themenentwicklung aus der Perspektive des "Issues-Managements“ (Ingenhoff und Röttger 2008) ist der Entwicklungsprozess nicht nur von Regierung und Opposition, sondern von allen Stakeholdern beeinflusst, die über diskursive Ressourcen verfügen.

Zusammengefasst geht das Konzept des Themenwettbewerbs davon aus, dass das Verhalten von Parteien im anhaltenden Wettkampf darüber, welche politische Themen auf der politischen Agenda ganz oben stehen, nicht nur durch Vermeidung, sondern auch durch aktive Auseinandersetzung und thematische Vernetzung miteinander gekennzeichnet ist (Spoon et al. 2014). 
Aus unserer skizzierten theoretischen Perspektive lassen sich mehrere Hypothesen ableiten:

1. Parteien werden durch die Agenda ihres Parteiensystems gezwungen, auch Themen zu adressieren, die für sie nicht vorteilhaft sind. Parteien reagieren verstärkt auf Themen, wenn andere Parteien diesen ebenfalls mehr Aufmerksamkeit schenken (Green-Pedersen und Mortensen 2015). Es folgt daraus die ParteiensystemAgenda-Hypothese:

H1 $_{1}$ Die Aufmerksamkeit, die eine Partei einem Thema schenkt, nimmt zu/ab, wenn sie insgesamt im Parteiensystem zu- oder abnimmt.

2. Parteien regieren auf Issue-Veränderungen nicht im gleichen Maße. So reagieren Parteien stärker auf saliente Themen, wenn der Eigentümer des Themas zum selben Parteienblock gehört bzw. ihr ideologisch nahesteht (Adams und SomerTopcu 2009; Green-Pedersen 2019). Diesbezüglich lässt sich folgende Parteienspektrums-Hypothese formulieren:

$\mathbf{H}_{2}$ Eine Partei reagiert stärker auf Issue-Änderungen, wenn ihr der Issue-Owner ideologisch nahesteht.

3. Solange ein Thema für Stimmgewinne wenig relevant ist - und daher scheinbar keine Salienz für den Wähler besitzt - ist eine Reaktion auf das von einer Partei besessene Thema durch parteipolitische Konkurrenten unwahrscheinlich. Die Reaktionsstärke hängt daher nicht nur von der ideologischen Distanz, sondern auch von der ,elektoralen Bedrohung“ ab, die von einer Partei ausgeht, die ein salientes Thema besitzt. Daraus folgt die Electoral-Threat-Hypothese:

$\mathbf{H}_{3}$ Je größer die Stimmanteile des Issue-Owners sind, desto wahrscheinlicher ist die Betonung des Themas durch andere Parteien.

4. „Mainstream-Parteien“ reagieren besonders stark auf Issue-Competition (GreenPedersen und Mortensen 2015; Spoon et al. 2014). Es handelt sich dabei um Parteien mit sehr hohem Stimmenanteil, die oft an Regierungen beteiligt sind (GreenPedersen und Mortensen 2015). Die höhere Anfälligkeit von Mainstreamparteien auf prominente, jedoch für sie ungünstigere Themen zu reagieren, wird dadurch erklärt, dass Regierungsparteien stärker von Änderungen der Agenda des Parteiensystems beeinflusst werden (Meguid 2005, 2008). Die Mainstream-Parteien-Hypothese lautet daher:

$\mathbf{H}_{4}$ Mainstream-Parteien zeigen im Vergleich zu Nischenparteien eine stärkere Reaktion auf Veränderungen der Agenda des Parteiensystems. 


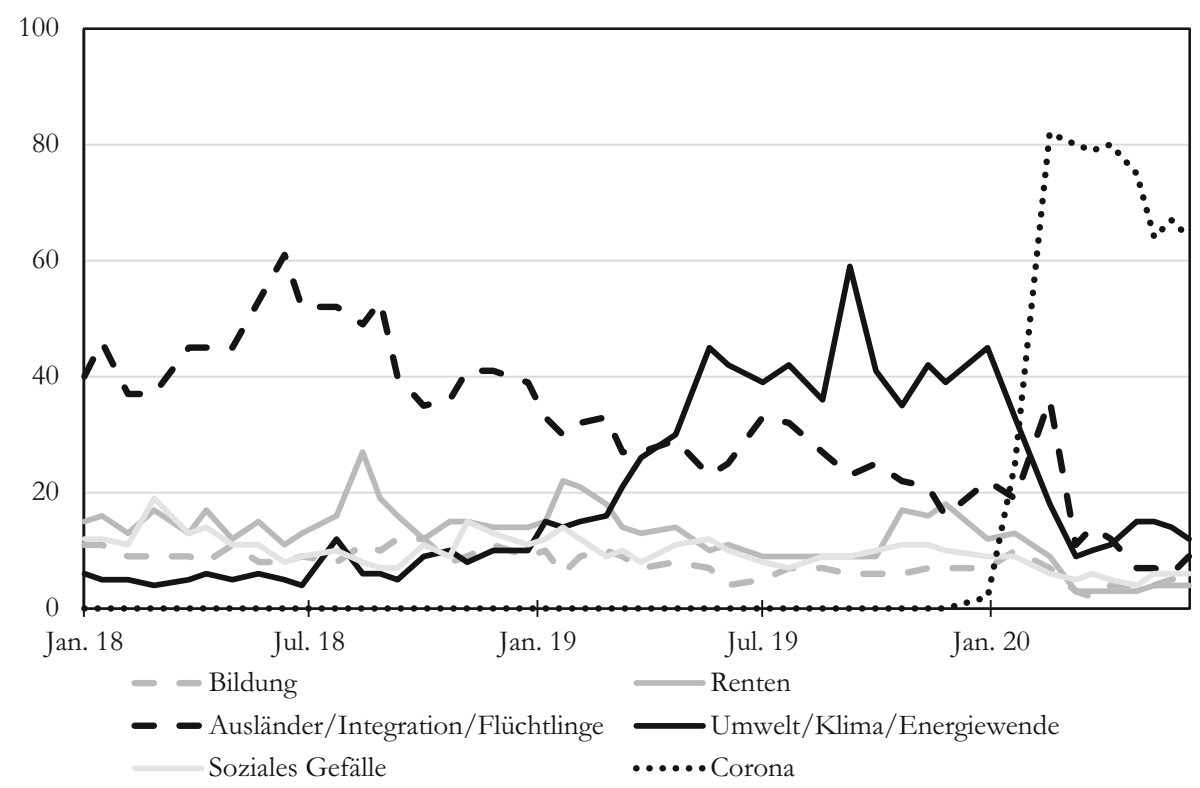

Abb. 2 Wichtige Probleme in Deutschland 2018-2020. (Eigene Darstellung nach Forschungsgruppe Wahlen 2020)

Alle vier Hypothesen lassen sich am bayerischen Themenwettbewerb der Jahre 2018 und 2019 auf qualitative Weise überprüfen. Hierzu muss geklärt werden, wie sich die Salienz des Umweltthemas entwickelte, wie Issue-Ownership und ideologische Nähe verteilt sind, und wie die Stimmanteile der konkurrierenden Parteien sich entwickelten.

Daten über die Themenentwicklung auf der politischen Agenda Bayerns liegen uns nicht vor, die auf das Bundesgebiet bezogenen Politbarometer-Umfragedaten bieten jedoch Näherungswerte. Abb. 2 zeigt die Nennungen der sechs wichtigsten Probleme in Deutschland von Anfang 2018 bis Mitte 2020. Das Top-Thema während des ganzen Jahres 2018 war Migration (Ausländer/Integration/Flüchtlinge), das jedoch im Frühjahr 2019 vom Themenfeld Umwelt/Klima/Energiewende überrundet wurde. Letzteres stieg in der zweiten Jahreshälfte zum dominierenden Thema auf. Beide thematischen frontrunner der Jahre 2018 und 2019 wurden jedoch Anfang 2020 vom neuen Top-Thema Corona regelrecht marginalisiert.

Die Ownership an den Themen Umwelt- und Klimapolitik konnten in den letzten Jahrzehnten ganz klar die Grünen behaupten. Viele Umfragen belegen, dass den Grünen dort die größte Kompetenz zugesprochen wird. Die Mainstream-Partei in Bayern ist zweifelsohne die CSU, die zwischen 1966 und 2008 sogar allein regierte.

Für die Messung der ideologischen Distanzen zwischen den Parteien können die Daten des Chapel Hill Expert Survey herangezogen werden, die sich auf umweltpolitische Einstellungen der Parteien beziehen (Polk et al. 2017). Auf der LinksRechts-Skala sind hierbei die Grünen bei 1,5 positioniert, die SPD bei 4,3, die CSU bei 6,2, die FDP bei 6,9 und die AfD bei 8,7. Die Freien Wähler (FW) rangieren wir, 
da im CHES nicht berücksichtigt, als liberal bis wertkonservative Partei, zwischen CSU und FDP ein. In der Umweltpolitik nehmen die FW keine eindeutige Haltung ein - so befürworten sie zum einen den Umstieg auf erneuerbare Energien, lehnen allerdings Dieselfahrverbote und den Bau von Stromtrassen ab.

\section{Diskursnetzwerkanalyse}

Wie neuere Anwendungen der Issue-Ownership und Issue-Competition-Theorien betonen, findet Themenwettbewerb hautsächlich über politische Kommunikation in den Massenmedien statt (Meyer et al. 2017). Der politische Kampf um Aufmerksamkeit als Teil des politischen Diskurses umfasst kommunikative Interaktionen zwischen politischen Akteuren über politische Probleme, Situationsdeutungen und Lösungsoptionen. Hierbei sind Diskursteilnehmer natürlich nicht auf politische Parteien bzw. politische Akteure im engeren Sinne beschränkt, sondern umfassen auch Akteure aus anderen gesellschaftlichen Teilsystemen wie Wirtschaft oder Wissenschaft. Diese Akteure tragen mit mündlichen (Wahlkampfreden, Interviews, etc.) als auch schriftlichen Beiträgen (Pressemitteilungen, Meinungsartikel, etc.) zum politischen Diskurs bei, indem sie sich für, oder gegen bestimmte Situationsdeutungen oder Politiken aussprechen. Solche Debatten finden in einer Vielzahl von „Diskursarenen"statt, von der Printpresse über das Fernsehen und den sozialen Netzwerken bis hin zu den Teilöffentlichkeiten der parlamentarischen oder zivilgesellschaftlichen Kommunikation.

Politische Diskurse sind einer formalen Analyse zugänglich und können als Netzwerke im Sinne der Sozialen Netzwerkanalyse (SNA) untersucht werden (Schneider et al. 2009; Leifeld 2016). Diskursteilnehmer werden dabei zu bestimmten Diskurselementen in Beziehung gestellt. Teilnehmende Akteure können zu bestimmten Aussagen in zustimmender oder ablehnender Weise Stellung nehmen (z. B. für oder gegen eine bestimmte Situationsdeutung oder eine bestimmte politische Maßnahme, etc.). Beziehen sich mehrere Diskursakteure auf identische Elemente oder Items, entsteht ein Netzwerk mit einer multiplen Anzahl von Knoten und Kanten. Solche Items können sich auf Beobachtungen erster Ordnung beziehen (z. B. eine bestimmte Problemwahrnehmung oder die Ankündigung einer Maßnahme), oder auf Beobachtungen zweiter Ordnung rekurrieren, in dem die Beobachtung erster Ordnung bestimmten wissenschaftlichen Konzepten zugeordnet werden (z. B. eine Problemdefinition als Ausdruck eines Frames, eines Überzeugungssystems, oder eines Narratives). In unserem Fall sind Statements von Diskursakteuren zu Einzelfragen die Beobachtung erster Ordnung, während die Zuordnung zu Themen die Beobachtungsebene zweiter Ordnung darstellt.

Die diskursiven Statements werden mit den Methoden der qualitativen Inhaltsanalyse kategorisiert, und diese Kategorien werden dann mittels numerischer Kodierung (binär oder kontinuierlich) mit Diskursakteuren in Beziehung gesetzt und mit formalen Methoden der Netzwerkforschung analysiert. Eine solche Analyse politischer Diskurse ermöglicht die Anwendung verschiedener Analyseverfahren, wobei besonders die Verfahren der Netzwerkvisualisierung im Vordergrund stehen (Brandes et al. 
1999). Unser diesbezügliches Vorgehen wird im Abschnitt zur Datenauswahl und -kodierung beschrieben.

Aus formaler Perspektive besteht ein Netzwerk aus einer Menge von Knoten, die mit einer Menge von Kanten verbunden sind. Im Unterschied zur One-ModeGrundversion eines sozialen Netzwerks, in der eine Menge von Akteuren $A$ untereinander verbunden sind, besteht ein Diskursnetzwerk im ersten Schritt aus einem Two-Mode-Netzwerk, das zwei unterschiedliche Knoten-Mengen mit Kanten verbindet: Akteure und Konzepte. Mittels Matrixmultiplikation ist es dann möglich, daraus One-Mode-Akteursnetzwerke als auch One-Mode-Konzeptnetzwerke abzuleiten (Newman 2010). Solche Akteursnetzwerke repräsentieren dann Beziehungen, die die Akteure über gemeinsame Konzepte verbinden, wobei solche Verbindungen auch Konkurrenzbeziehungen sein können. Konzeptnetzwerke repräsentieren Beziehungen, die Konzepte über gemeinsame Akteure verbinden.

\subsection{Datenauswahl und -kodierung}

Die hier analysierte Diskursarena umfasst keine gesamte Legislaturperiode, aber die Diskursentwicklung entlang dreier Schlüsselmomente: der Landtagswahl im Oktober 2018, dem Volksbegehren im Februar 2019 und der Wahl zum Europäischen Parlament im Mai 2019. Unser Untersuchungszeitraum erstreckt sich vom 03.09.2018 bis zum 20.09.2019: Vom Auftakt des bayrischen Landtagswahlkampfes beim Gillamoos-Volksfest am 03.09.2018 bis zum Tag des Klimagipfels am 20.09.2019, an welchem das Klimapaket der Bundesregierung auf den Weg gebracht wurde.

Die Textgrundlage der Analyse bilden Artikeln der Süddeutschen Zeitung (SZ) aus diesem Zeitraum, in denen sich bayrische Diskursakteure namentlich zu den Themen Umwelt- und Klimaschutz positionierten. Die Verwendung von Zeitungartikeln hat dabei den entscheidenden Vorteil, dass im Gegensatz zu anderen häufig in der Issue-Forschung verwendeten Datenquellen wie Wahl- oder Grundsatzprogrammen zeitlich präzise Diskursdynamiken aufgezeigt werden können (Seeberg 2020). Die Beschränkung auf eine einzige Datenquelle birgt natürlich einen gewissen Bias, jedoch bietet die SZ als Qualitätsmedium mit breiter lokaler Berichterstattung über Bayern einen durchaus geeigneten Ausgangspunkt. Zudem wurden die Daten im Rahmen einer studentischen Abschlussarbeit erhoben, und sind dadurch in ihrem Umfang begrenzt. Nichtdestotrotz machen die erhobenen Daten es durchaus möglich, das Potenzial unseres analytischen Ansatzes zu demonstrieren und zu illustrieren.

Die Auswahl der für die Kodierung in Frage kommenden Artikel erfolgte in einem zweistufigen Prozess. Zuerst wurde das Onlinearchiv der SZ mit dem Suchbegriff (umwelt* OR klima* OR Fridays for Future) AND (bayer*) durchsucht. Da im gewählten Zeitraum 4127 Treffer gefunden wurden, wurden die Filtereinstellungen präzisiert: So wurden nur Artikeln aus der Druckausgabe der SZ berücksichtigt, und Beilagen, Landkreisausgaben und überregionale Extras ausgeschlossen. Außerdem wurden irrelevante Rubriken wie etwa Leserbriefe oder Kulturbeiträge herausgefiltert, wobei insgesamt 234 von 293 Artikeltypen für die Suche zugelassen wurden. Dadurch ergab sich eine Suchmenge von 316 Artikeln, welche in einem zweiten Schritt manuell gesichtet wurden. Alle Artikel, bei denen Statements sich nicht ex- 
plizit einer Person zuordnen ließen, sowie solche, die aufgrund des offen gefassten Suchbegriffes inhaltlich nicht relevant waren, wurden aussortiert. Durch diesen Prozess konnten final 52 Artikel als relevant identifiziert werden. Die ausgewählten Artikel wurden mit dem „Discourse Network Analyzer“ (dna) von Philipp Leifeld (2019, 2012) manuell kodiert, wobei jedem Statement vier Variablen zugeordnet wurden: Die Person, von der das Statement stammt, die dazugehörige Organisation, das Konzept, zu dem die Äußerung thematisch eingeordnet werden konnte, und schließlich ob der Akteur sich zustimmend oder ablehnend zu dem Konzept äußerte (Dummy-Kodierung). Die Kategorisierung erfolgte in einem induktiven Prozess über mehrere Anpassungsdurchläufe (Leifeld 2016). Um verschiedene Definitionen von Issues in der Analyse zu berücksichtigen, wurden den Statements Konzepte zugeordnet, die entweder als Politikziele, Politikmaßnahmen oder Problemdiagnosen kategorisiert werden konnten (Guinaudeau und Persico 2014).

Der Gesamtdatensatz enthält nach der beschriebenen Erhebung 244 Statements zu 86 verschiedenen Konzepten, welche von 73 Personen aus 19 Organisationen in insgesamt 52 Artikeln getätigt wurden. Für die verschiedenen Schritte unserer Analyse wurden entsprechende Teildatensätze erzeugt. Für die Visualisierung der Diskursnetzwerke wurde die Software ,visone“ (Brandes und Wagner 2019) verwendet.

\section{Ergebnisse}

Unsere Analyse folgt mehreren Schritten: Zunächst wird der oben erläuterte Gesamtdatensatz verwendet, um einen Überblick über die Salienz der umweltpolitischen Themen zu gewinnen. Anschließend betrachten wir Statements aller bayerischen Parteien und einer Auswahl wichtiger gesellschaftlicher Organisationen und deren Zentralität im Diskurs in den jeweiligen Zeitabschnitten. In einem weiteren Analyseschritt werden die Statements zu Policy-Positionen in Themenbereichen gruppiert und einer Zentralitätsanalyse der Diskursthemen unterworfen. Abschließend erzeugen wir Akteursnetzwerke als One-Mode-Projektionen mittels Matrixmultiplikation, die aufzeigen, welche Akteure um die gleichen Themen konkurrieren.

\subsection{Salienz und Diskursbeteiligung}

Um die Entwicklung des Diskurses netzwerkanalytisch vergleichen zu können, wurde der Untersuchungszeitraum in einzelne Phasen unterteilt:

Phase 1 03.09.2018-30.01.2019: Wahlkampf, Landtagswahl - Start Volksbegehren

Phase 2 31.01.2019-26.05.2019: Volksbegehren - Europawahl

Phase 3 27.05.2019-20.09.2019: Post-Europawahl - Klimagipfel Berlin Zunächst interessiert uns die Salienz der Themen im Zeitablauf, die in Abb. 3a dargestellt ist. Deutlich erkennbar ist dabei die zunehmende Salienz im Zuge des 

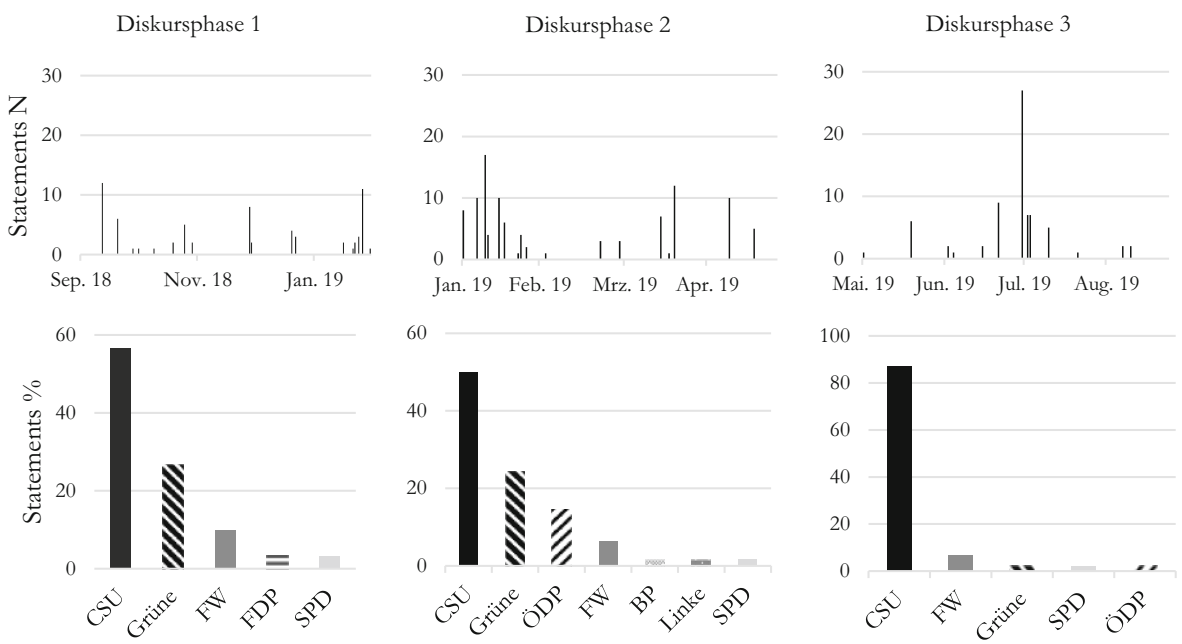

Abb. 3 a Salienz der Themen in den drei Diskursphasen (absolute Diskursbeiträge), b Diskursbeteiligung der bayrischen Parteien in den drei Diskursphasen (prozentuale Diskursbeiträge)

Volksbegehrens Ende Januar/Anfang Februar. Wie auch vor der Landtagswahl lässt sich vor der Europawahl keine Intensivierung des Diskurses feststellen, dafür zeigt sich in der dritten Diskursphase (besonders Ende Juli) klar das umweltpolitische Feuerwerk der CSU.

Ein ebenfalls interessanter Aspekt des politischen Wettbewerbs ist die Diskursbeteiligung, wobei in dieser Analyse alle Statements gezählt werden, egal ob sie zustimmender oder ablehnender Natur sind. Abb. 3b zeigt die Varianz der Parteibeteiligung in den jeweiligen Diskursphasen. Hier wiederholt sich das eben beschriebene Muster in wesentlichen Zügen: Durch das Volksbegehren diversifiziert sich der Diskurs und damit auch die Zahl der engagierten Parteien in der zweiten Diskursphase, wohingegen in der dritten Diskursphase die CSU ihre parteipolitischen Konkurrenten ganz klar dominiert und einen Diskursanteil von fast $87 \%$ erreicht. Interessanterweise tritt sich damit augenscheinlich durchgehend als aktivster parteipolitischer Akteur im Bereich Umwelt- und Klimaschutz auf, was sich in der ersten Diskursphase jedoch vor allem auch auf ablehnende Policy-Positionen gründet, wie wir in der nachfolgenden Analyse zeigen.

\subsection{Vergleich der Diskursentwicklung in drei Phasen}

Im folgenden Abschnitt wird die Diskursentwicklung mittels einer Zentralitätsanalyse der Diskursphasen nachgezeichnet. Alle Statements wurden hier nach PolicyPositionen gruppiert, die auf dem Rand der Zielscheibendiagramme abgetragen werden. Die Akteure werden alsdann entsprechend der Summe ihrer Statements auf den verschiedenen Ringen eines Zielscheibendiagramms positioniert (Gradzentralität). Die Summe ergibt sich als Addition zustimmender (grüne Verbindungen) und Subtraktion ablehnender (rote Verbindungen) Positionen. Wie Abb. 4 zeigt, nehmen in der ersten Diskursphase die Grünen und die Umweltverbände die zentralsten Posi- 


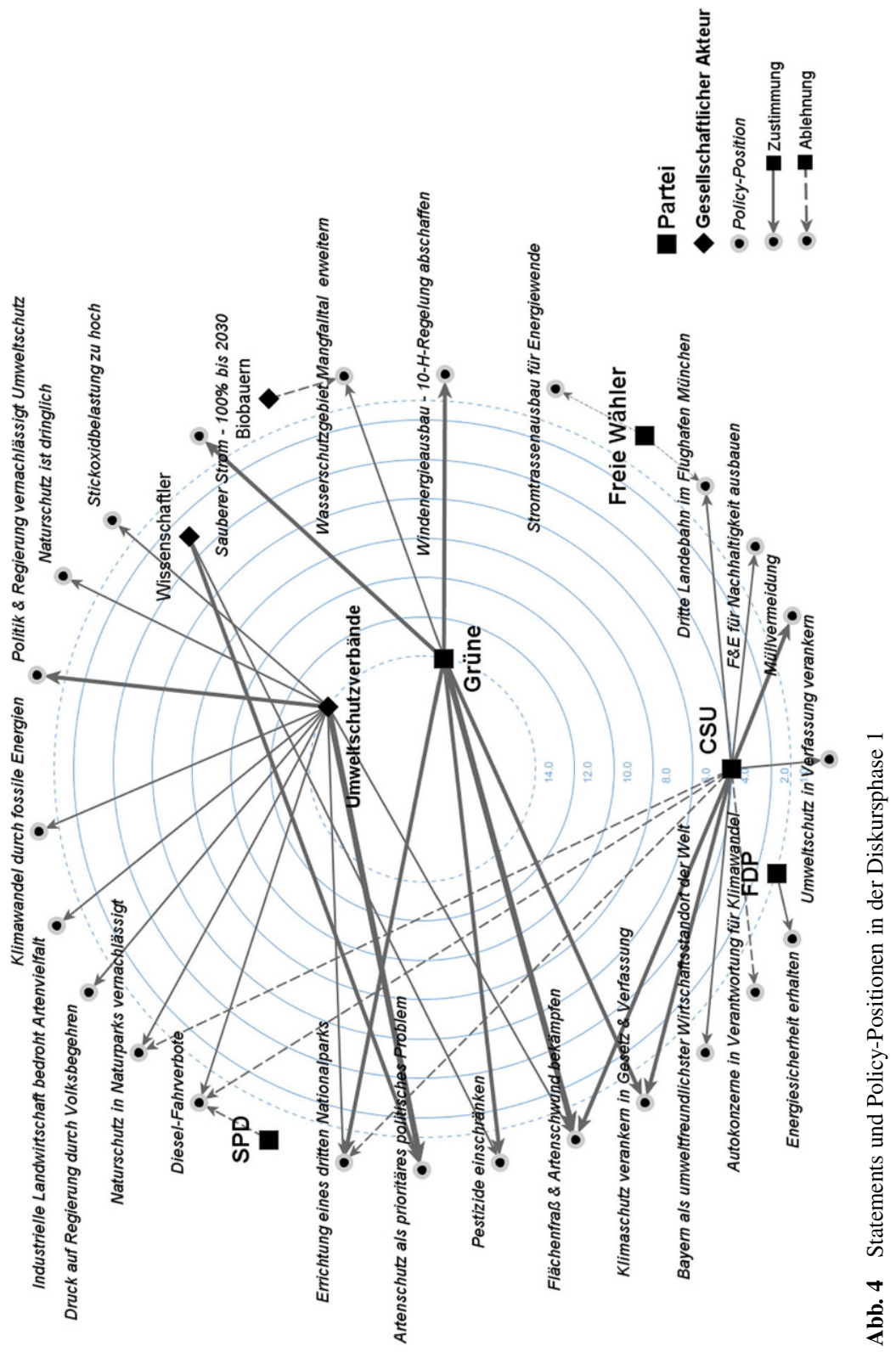

tionen ein, während die CSU eher peripher aber noch zentraler als die Freien Wähler positioniert ist. Ganz am Rand halten sich SPD und FDP auf.

In der zweiten Diskursphase rücken umwelt- und klimapolitische Themen durch das Volksbegehren klar in den medialen und politischen Fokus. Die CSU reagiert äußerst schnell auf die deutlich gestiegene Salienz des Themas und reagiert nicht 

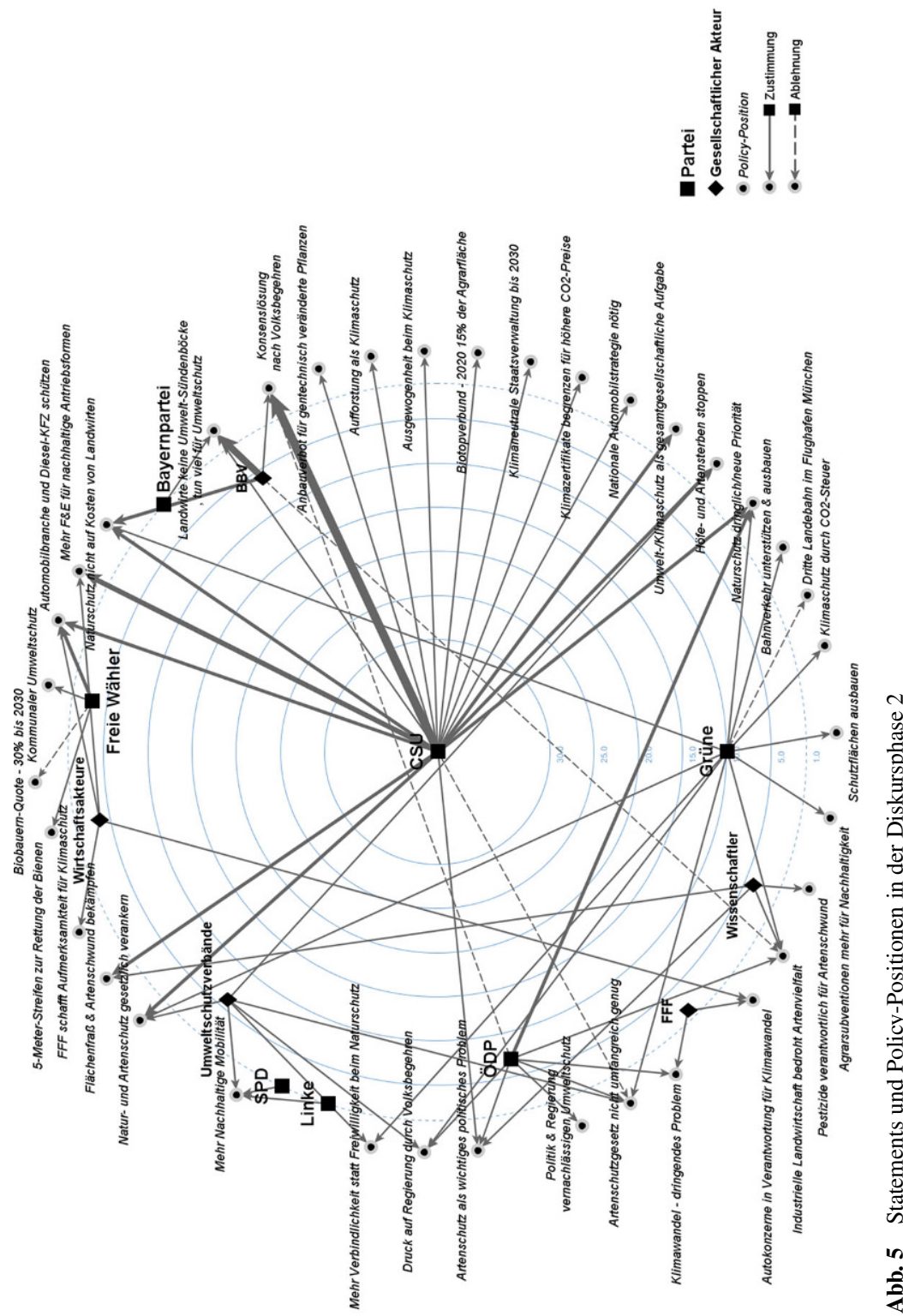

nur mit der Betonung ihrer bisherigen umweltpolitischen Leistungen, sondern auch mit einem wahren Feuerwerk an ökologischen Ankündigungen (Abb. 5).

Die Wirkungen dieser diskursiven Interventionen zeigen sich in Abb. 6, welche die dritte Diskursphase umfasst. Hier etabliert sich die CSU noch klarer im Zentrum des Diskurses. Selbst die Grünen, die ÖPD und die Umweltverbände sind nun peripher positioniert, so massiv ist die Attacke der CSU auf das umweltpolitische Themenfeld. 


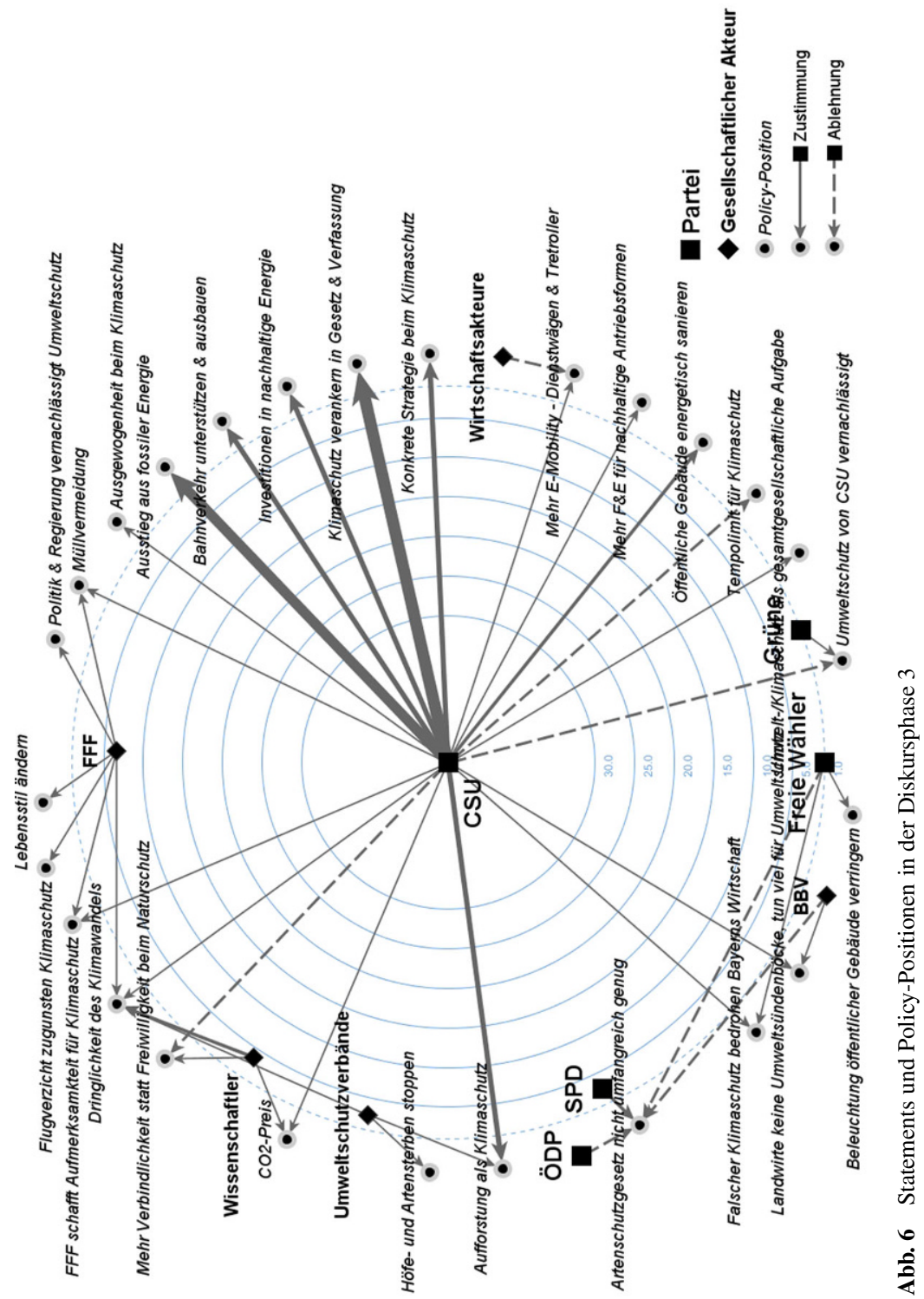

Dass die CSU so stark im Zentrum steht und die übrigen Akteure weitgehend marginalisiert bleiben, liegt zweifelsohne auch an der oben erwähnten Selektivität des inhaltsanalytischen Vorgehens. Nichtsdestotrotz kann die Analyse ganz offenkundig die Wirksamkeit des „Issues-Managements“ der CSU (und insbesondere ihres Ministerpräsidenten) aufzeigen. 


\subsection{Themenfelder}

Der nächste Schritt der Analyse besteht in der Zuordnung der einzelnen PolicyPositionen zu Themenfeldern, sowie der Darstellung der Zentralität der jeweiligen Themenkategorien in Zielscheibendiagrammen. Die Aufteilung orientiert sich dabei weitgehend an Jänicke (2006). In diesem Schritt beschränken wir die Analyse auf die Positionen der Parteien. Auch hier wurde als Zentralitätsmaß der Außengrad gewählt, der die Anzahl der Policy-Positionen angibt, die eine Partei in einem Themenfeld adressiert. Im Unterschied zur vorausgehenden Analyse entspricht die Positionierung im Zielscheibendiagramm als auch die Dicke der Verbindungen nicht der Summe positiver abzüglich negativer Beziehungen einer Partei, sondern der Summe der Häufigkeiten der Policy-Positionen gemessen als absolute Beträge. Die Themenzentralität in den drei Diskursphasen gibt Abb. 7 wieder.

In der ersten Phase lässt sich noch keine Diskursfokussierung auf einen alleinigen Themenkomplex feststellen. Die Debatte ist vor allem durch Themen geprägt, die bereits länger auf der umweltpolitischen Agenda in Bayern stehen (Kohout 2013), und Klimapolitik nimmt eine eher semiperiphere Rolle ein. Die Hauptdiskutanten im Parteienspektrum sind die CSU und die Grünen. In der zweiten Diskursphase zeigt sich klar die Bedeutung des Volksbegehrens: Der Themenkomplex Natur- \& Artenschutz nimmt ganz deutlich die zentralste Position ein, und wird neben CSU und Grünen auch von der ÖDP als außerparlamentarische Initiatorin des Volksbe-

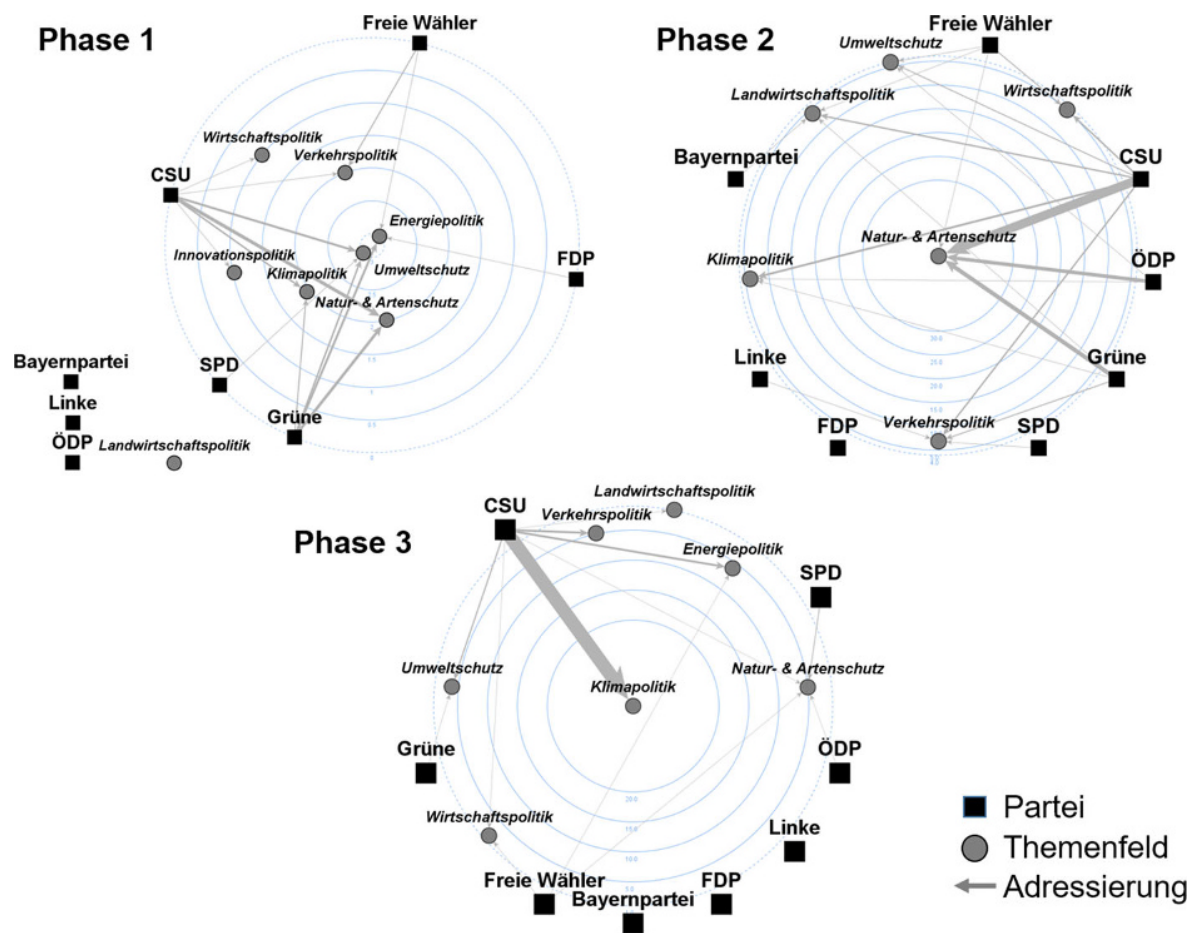

Abb. 7 Die Adressierung von Themenfeldern durch die bayrischen Parteien 
gehrens adressiert. Interessant ist, dass die CSU jedoch gleichzeitig innerhalb des Umweltdiskurses auch einen wirtschafts-, verkehrs-, und landwirtschaftspolitischen Fokus verfolgt, und sich etwa bemüht, den Schutz von „Bauern und Bienen“ als Ziel zu kommunizieren. Nach der Europawahl, in der dritten Phase, verdrängt der Klimaschutz alle bisherigen Themen an den Rand des Diskurses. Dabei wird er jedoch ausschließlich von der CSU thematisiert, während sich etwa SPD und ÖDP weiterhin mit dem Natur- und Artenschutz und der Nachwirkung des Volksbegehrens beschäftigen.

Die Analyse der zentralen Themen hat damit eine deutliche Verschiebung der Diskursfoci in den unterschiedlichen Zeitfenstern gezeigt. Es lässt sich festhalten, dass beginnend mit „Rettet die Bienen“ eine Neuausrichtung und Fokussierung des Diskurses auf Natur- und Artenschutz stattfand. Im Sommer 2019 wiederum lässt sich ein sehr starker Fokus auf den Themenbereich Klimaschutz erkennen, der zweifelsohne durch den allgemeinen Aufschwung des Themas in Deutschland geprägt ist, aber sicherlich auch als Konsequenz aus der hohen Beteiligung am Volksbegehren gewertet werden kann. Insbesondere die CSU adressiert mit einem breiten Spektrum von Policy-Positionierungen dieses Themenfeld und übernimmt augenscheinlich in Bayern die komplette Issue-Ownership.

\subsection{Themenwettbewerb der Parteien in der Gesamtbetrachtung}

Dies führt zu unserem letzten Analyseschritt, in dem wir ein Gesamtbild des Themenwettbewerbs in Bayern skizzieren. Parteienwettbewerb findet auf mehreren Ebenen statt: Durch die allgemeine ideologische Ausrichtung im politischen Raum, durch die Attraktivität von Kandidaten, und schließlich durch die den Parteien zugeschriebenen Problemlösungskompetenzen. Unser letzter Analyseschritt zielt nun auf ein integriertes Gesamtbild über die Diskursphasen hinweg, das zusammenfasst, welche Wettbewerbsbeziehungen auf dieser thematischen Ebene existierten, indem Parteien dieselben Themenbereiche mit Diskursbeiträgen adressieren (Abb. 8).

Für diese Analyse werden die Themen-Affiliationsmatrizen aller drei Zeiträume aufsummiert, und aus dieser Ergebnismatrix wird eine One-Mode-Projektion mittels

Abb. 8 Thematischer Wettbewerb 2018 und 2019 in Bayern im Bereich der Umweltpolitik

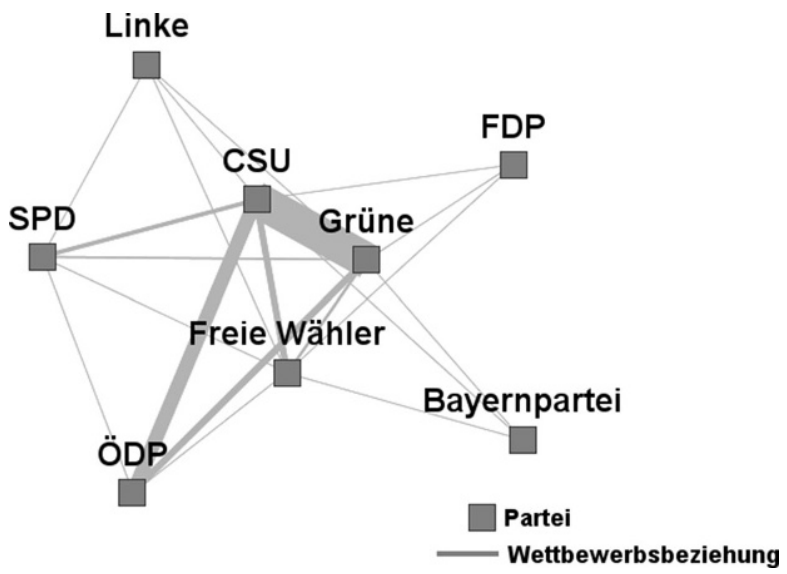


Matrixmultiplikation erzeugt. Das Ergebnis ist eine Matrix von Wettbewerbsbeziehung zwischen den Parteien, die im Schaubild als Graph mit Visones Stress-Layout visualisiert ist (Brandes 2008). In diesem nehmen die mittig positionierten Akteure zentrale Positionen im Diskurs ein. Die Breite der Linien verweist jeweils auf die Häufigkeit, mit der Diskursakteure durch Diskursbeiträge dieselben Themen adressieren und hiermit in einem Wettbewerbsverhältnis stehen. Klar ersichtlich wird dadurch, dass CSU und Grüne im untersuchten Diskurs Hauptgegner füreinander sind. Weitere Konkurrenten der CSU im Themenkampf sind die ÖDP und ihr Koalitionspartner, die Freien Wähler.

\subsection{Ergebniszusammenfassung}

Die durch die beiden Theorien gestützten Vermutungen, die in den vier Hypothesen in Abschn. 3 formuliert sind, können insgesamt nur teilweise bestätigt werden was jedoch gerade die interessante Dynamik des Themenwettbewerbs in Bayern hervorhebt. Insgesamt hat die zunehmende Salienz der Umwelt- und Klimapolitik besonders im Jahre 2019 die Aufmerksamkeit für dieses Thema gestärkt. Es konnte jedoch eine starke Variation in der Aufmerksamkeit für grüne Themen zwischen den Parteien beobachtet werden, eine wie in unserer ersten Hypothese vermutete Parallelität konnte nicht bestätigt werden. Einzig die Reaktionen von CSU, Grünen und ÖDP auf „Rettet die Bienen“ könnten als eine solche Entwicklung angesehen werden, SPD, FW, FDP und auch AfD spielen hingegen im Diskurs nur eine geringe bis keine Rolle. Dadurch konnte auch die Parteienspektrum-Hypothese in diesem Fall nicht bestätigt werden. Es konnte nicht festgestellt werden, dass ideologisch näher an den Grünen einzuordnende Parteien sich im Zuge einer Salienz des Themas ebenfalls stärker in den umweltpolitischen Diskurs eingebracht hätten. Für ein systematische Überprüfung dieser Parteienspektrum-Hypothese wäre es letztlich nötig, mehrere bzw. viele Parteienspektren miteinander zu vergleichen, was in dieser fallmäßig beschränkten und qualitativen Analyse nicht möglich ist.

Die Beantwortung der Electoral-Threat-Hypothese fällt wiederum uneindeutiger aus: Nach den Landtagswahlen 2018, bei denen die bayrischen Grünen einen enormen Stimmenzuwachs verzeichneten, konnte bis zum erfolgreichen Volksbegehren keine Zunahme an umweltpolitischen Äußerungen durch die politischen Gegner, auch die CSU, verzeichnet werden. Die plötzliche Aufnahme zahlreicher klimapolitischer Forderungen in das Diskursrepertoire der CSU lässt sich hingegen durchaus als eine Reaktion auf das wiederholt starke Abschneiden der Grünen bei den Europawahlen werten. Insgesamt bestätigt das Verhalten der CSU unsere vierte Hypothese: Obwohl unsere Mainstreampartei, die CSU, eine große ideologische Distanz zum Issue-Eigentümer ökologischer Themen aufweist, verstärkte sie die Ansprache grüner Themen deutlich. Als Mainstream-Partei ist sie gezwungen, sich auch in diesem für sie ungünstigen Themenbereich zu engagieren. Interessant ist, dass das IssueManagement der bayerischen Staatsregierung nicht nur Eindämmungspolitik in dem Sinne betrieb, dass man einfach nur die eigene umweltpolitische Performanz auf den Scheffel stellte oder versuchte, den Diskurs durch geschickte Neudefinition der Problemstellung gewinnbringend umzudeuten (Holian 2004), sondern im Sinne einer Vorwärtsverteidigung auf Attacke schaltete, und das Themenfeld mit einem 
ganzen Spektrum von medienwirksamen Maßnahmenankündigungen bombardierte. Im November 2019 verabschiedete Söders Kabinett das erste Bayerische Klimaschutzgesetz, welches von einer Klimaoffensive mit fast hundert Einzelmaßnahmen begleitet wurde (Bayrische Staatsregierung 2019).

\section{Diskussion}

Die Analyse des umweltpolitischen Diskurses hat gezeigt, dass einzig die CSU (neben den Issue-Ownern) auf die zunehmende Salienz der Themen Umwelt- und Klimaschutz reagierte. Die Gründe dafür möchten wir an dieser Stelle noch genauer beleuchten. Zum einen zeigt die Reaktion der CSU klar, dass Mainstreamparteien einem starken Zwang ausgesetzt sind, auf die obersten Themen auf der Agenda des politischen Diskurses zu reagieren. ÖDP und Grünen gelang es durch das überaus erfolgreiche Volksbegehren ihre Themen so dominant auf dieser Agenda zu positionieren, dass die CSU schlichtweg gezwungen war, darauf zu reagieren. Dies demonstriert eindrücklich die Agendasetzermacht von Nischen- und Oppositionsparteien. Zum anderen verdeutlicht unsere Analyse auch, dass Mainstreamparteien bei ausreichend hohen Stimmgewinnen ihrer politischen Gegner durchaus auch Themen adressieren, die für sie ideologisch weniger naheliegend sind. Die Reaktionsgeschwindigkeit und -stärke hängt dabei sicherlich auch vom strategischen Gespür der Parteien ab.

Besonders Markus Söders scheint immer schon ein großes Talent besessen zu haben, auf aktuell wichtige Themen schnell und entschieden zu reagieren und so Entscheidungs- und Handlungsfähigkeit zu demonstrieren. Dies hatte sich z. B. nach der Atomkatastrophe in Fukushima gezeigt, nach welcher sich der damalige Umweltminister als einer der ersten bayrischen Politiker klar für eine Abkehr von der bisherigen Atompolitik aussprach (Tratzmiller 2018). Mit Sicherheit kann gesagt werden, dass der Ministerpräsident nach den Stimmverlusten bei der Landtagswahl 2018 und insbesondere auch der enormen Unterstützung des Volksbegehrens gelernt hat. Umweltpolitik hat sich zu einem Themenkomplex entwickelt, der für große Teile der Bevölkerung relevant ist und daher aktiv adressiert werden muss. Söder verwandelte sich daher in bemerkenswert kurzer Zeit vom „Spalter der Nation zu Bayerns oberstem Umweltpolitiker“ (Clauß 2019).

Die Frage, ob Söder und die CSU durch die Repositionierung im umweltpolitischen Diskurs allerdings auch erfolgreich einen Zuwachs an dauerhafter IssueOwnership erzielen konnten, stellt sich durchaus. Es könnte sein, dass die Wähler die diskursiven Aktivitäten der CSU als pures Issue-Management durchschauen und viele der Maßnahmen als greenwashing oder rebranding abhaken. Es gibt jedoch Anzeichen, dass diese Themeninterventionen Söders sich in der Bevölkerung verfangen. Zwar liegen Umfragen zur Umweltkompetenz in Bayern nicht vor, jedoch zeigt der Bayern-Monitor der Augsburger Allgemeinen, dass die Beliebtheit Söders zwischen Juli und September 2019 anstieg und die Anzahl der unzufriedenen Bürger abnahm (Liermann 2019). Seine zunehmende Beliebtheit verdankte Söder dabei jedoch vor allem Wählern der CSU, in der Wählerschaft der Grünen war Stand September 2019 nur ein gutes Fünftel mit seiner Arbeit zufrieden (Liermann 2019). Dies 
wäre ein Indiz, dass die Mehrheit der Bürger seine umwelt- und klimapolitischen Bemühungen als eher unglaubhaft einschätzte. Nichtdestotrotz genoss Söder durch diesen Aufbau eines Macherimages in Verbindung mit einer ausgeprägten Responsivität auf saliente Themen eine anhaltend hohe Beliebtheit in der gesamtdeutschen Bevölkerung und wurde im Vorfeld der Bundestagswahl 2021 sogar als potenziell aussichtreicher Kanzlerkandidat der Union gehandelt.

$\mathrm{Zu}$ guter Letzt müssen noch einige Beschränkungen unseres Vorgehens angesprochen werden. Aufgrund des beschränkten Umfanges dieser Analyse wurde eine unitary actor assumption getroffen, und der Diskurs nicht noch innerhalb der Parteiebene differenziert dargestellt (vgl. Bhattacharya 2020). In der Tat zeigen unsere Daten, dass etwa die umfassenden umweltpolitischen Forderungen der CSU fast ausschließlich auf Markus Söder zurück gehen. Jedoch traten auch kaum CSUAkteure mit widersprüchlichen Statements in Erscheinung, weshalb wir dies als $\mathrm{Zu}$ stimmung für das Söderschen Issue-Management werten. Grundsätzlich bieten die erhobenen Daten aber die Möglichkeit innerparteiische Diskurse zu analysieren und zu vergleichen, was erneut die vielfältigen Anwendungspotenziale unserer Methode unterstreicht.

Eine weitere Einschränkung ist, dass die rudimentäre Abbildung des Europawahlkampfes möglicherweise auf den verwendeten Suchbegriff zurückzuführen ist. So wurden durch die Bedingung, dass eine Variante von bayer* im Artikel enthalten sein musste, eventuell Statements zur Umweltpolitik nicht aufgefunden, da Akteure im europäischen Kontext eher Forderungen ohne Nennung Bayerns äußerten. Darüber hinaus wirkt die Beschränkung auf Zeitungsartikel immer selektiv auf den Gesamtdiskurs (Nagel 2016). Eine ergänzende Analyse von Positionspapieren, Wahlprogrammen und Parlamentsdebatten würde sicher die Vielfalt an parteipolitischen Akteuren und Konzepten erhöhen. Auch würde die Miteinbeziehung von weiteren - insbesondere regionalen - Zeitungen zu einer größeren Bandbreite der beobachteten Berichterstattung beitragen. All dies bietet Ansatzpunkte für zukünftige Forschung.

\section{Konklusion}

Auch wenn unser Datensatz aus forschungspragmatischen Gründen beschränkt ist und gewisse Selektivitäten aufweist, so konnten wir mit unserer Analyse demonstrieren, dass die recht neue Methode der Diskursnetzwerkanalyse auf fruchtbare Weise in der Issue-Competition-Forschung angewandt werden kann. Wir konnten zeigen, dass sich die Beziehungen zwischen Parteien und politischen Themen über diskursive Statements messen und als Netzwerke darstellen lassen, die sich mit verschiedensten Methoden der Netzwerkforschung präzise analysieren lassen. Hierbei konzentrierten wir uns auf verschiedene Verfahren der Netzwerkvisualisierung, bei denen wir insbesondere die Zentralitätsanalyse einsetzten. Das gesamte Potenzial, das in der Verbindung von Netzwerk und Themenanalyse steckt, konnten wir daher nur ansatzweise aufzeigen. Über die von uns verwendeten Methoden hinaus, könnten auch Verfahren der Cliquen-, Cluster- und Blockmodellanalyse herangezogen werden, um Themencluster systematisch herauszuarbeiten und Themenblöcke 
zu aggregieren. Schließlich haben sich in den letzten Jahrzehnten auch spezifische Verfahren der Netzwerkregression entwickelt, die eine Überprüfung von Hypothesen mittels teststatistischer Methoden ermöglichen. Hierbei werden jedoch umfangreiche Daten - zum Beispiel mehrere vergleichende Analysen - vorausgesetzt, die in unserem Fall aus den dargelegten Gründen nicht vorliegen. Wir haben jedoch die Hauptmethoden präsentiert, die in den letzten Jahren in international Top-Zeitschriften publiziert wurden.

Diskursnetzwerkanalyse als Kombination von Netzwerkforschung und qualitativer Inhaltsanalyse hat sich mit seiner spezialisierten Software (Leifeld 2019) generell zu einem fruchtbaren Instrument der Medienforschung entwickelt, um Beziehungen zwischen Diskursakteuren und Diskursstatements und deren Interaktion im gesamten Medienspektrum abzubilden. Wie in vielen anderen Analysen dieser neuen Methode haben wir uns dabei auf Presseartikel und hierbei natürlich nur auf eine Diskursarena konzentriert. Inzwischen gibt es auch viele Analysen im Bereich der Sozialen Medien oder der Blogosphäre, in denen bislang zwar Themennetzwerke identifiziert, aber nicht aus der Perspektive des Themenwettbewerbs betrachtet wurden. Ein gutes Beispiel stellt die Untersuchung von Maier et al. (2018) dar, wo Themennetzwerke aus der Perspektive kommunikationswissenschaftlicher Theorien und Modelle untersucht werden.

Ganz generell bietet die Verknüpfung von Netzwerkanalyse und Inhaltsanalyse aus vergleichender Perspektive den Vorteil, dass Themenkonjunkturen auch hochauflösend longitudinal untersucht werden können. Über exakte Zeitfenster kann die Dynamik des Themenwettbewerbs und die Interaktion der Parteien im Diskurs detailliert verfolgt werden. Die Auswirkung von Salienzveränderungen durch unterschiedliche Schlüsselereignisse kann so in hoher Granularität herausgearbeitet und modelliert werden.

Am Beispiel der bayrischen CSU haben wir so das Wettbewerbsverhalten einer Volkspartei im Kampf um Issue-Ownership grüner Themen illustriert. Ob die CSU dem Klima- und Umweltschutz dauerhaft eine Spitzenposition in ihrem politischen Programm zukommen lassen wird, bleibt weiterhin fraglich. Sie hat jedoch verstanden, dass sie - wenn sie weiterhin dem Anspruch einer Volkspartei genügen will um die Ansprache „grüne Themen“ nicht herumkommen wird.

Interessant ist und bleibt außerdem der bereits in Abb. 2 gezeigte Einfluss der Corona-Pandemie auf die Entwicklung dieses Themenwettbewerbs. In kürzester Zeit wurde die Umwelt- und Klimapolitik wieder auf die unteren Plätze verwiesen. Deutlich wird hier sichtbar, dass der Themenwettbewerb ein äußerst volatiler Prozess ist, dessen weitere intensive Analyse gerade auch mit der Methode der Diskursnetzwerkanalyse äußerst vielversprechend erscheint.

Danksagung Eine frühere Version dieses Artikels wurde auf dem Workshop „Parteiendifferenz in der Umweltpolitik“ an der Universität Göttingen vorgestellt. Wir danken den Teilnehmer*innen sowie den drei anonymen Gutachter*innen für ihre hilfreichen Kommentare.

Funding Open Access funding enabled and organized by Projekt DEAL.

Open Access Dieser Artikel wird unter der Creative Commons Namensnennung 4.0 International Lizenz veröffentlicht, welche die Nutzung, Vervielfältigung, Bearbeitung, Verbreitung und Wiedergabe in jeglichem Medium und Format erlaubt, sofern Sie den/die ursprünglichen Autor(en) und die Quelle ord- 
nungsgemäß nennen, einen Link zur Creative Commons Lizenz beifügen und angeben, ob Änderungen vorgenommen wurden.

Die in diesem Artikel enthaltenen Bilder und sonstiges Drittmaterial unterliegen ebenfalls der genannten Creative Commons Lizenz, sofern sich aus der Abbildungslegende nichts anderes ergibt. Sofern das betreffende Material nicht unter der genannten Creative Commons Lizenz steht und die betreffende Handlung nicht nach gesetzlichen Vorschriften erlaubt ist, ist für die oben aufgeführten Weiterverwendungen des Materials die Einwilligung des jeweiligen Rechteinhabers einzuholen.

Weitere Details zur Lizenz entnehmen Sie bitte der Lizenzinformation auf http://creativecommons.org/ licenses/by/4.0/deed.de.

Interessenkonflikt G.-J. Westenberger und V. Schneider geben an, dass kein Interessenkonflikt besteht.

\section{Literatur}

Adams, James, und Zeynep Somer-Topcu. 2009. Policy adjustment by parties in response to rival parties' policy shifts: spatial theory and the dynamics of party competition in twenty-five postwar democracies. British Journal of Political Science 39(4):825-846. https://doi.org/10.1017/ S0007123409000635.

Bauchmüller, Michael. 2019. Söders grünes Feuerwerk: Klimaschutz. https://www.sueddeutsche.de/ politik/klimaschutz-markus-soeder-csu-1.4544542. Zugegriffen: 15. Jan. 2020.

Bayrische Staatsregierung. 2019. Bericht aus der Kabinettssitzung vom 19. November 2019. https://www. bayern.de/bericht-aus-der-kabinettssitzung-vom-19-november-2019/. Zugegriffen: 03. Febr. 2020.

Bayrischer Landtag. 2018. Das amtliche Endergebnis. https://www.bayern.landtag.de/abgeordnete/ landtagswahl-2018/das-amtliche-endergebnis/. Zugegriffen: 25. Febr. 2020.

Bayrisches Landesamt für Statistik. 2019. Volksbegehren „Artenvielfalt \& Naturschönheit in Bayern“. https://www.statistik.bayern.de/presse/mitteilungen/2019/pm03/index.html. Zugegriffen: 25. Febr. 2020.

Bayrisches Staatsministerium für Umwelt und Verbraucherschutz. 2020. Ein halbes Jahrhundert Bewahrung unserer Lebensgrundlagen: das Bayerische Umweltministerium. https://www.stmuv.bayern.de/ ministerium/aufgaben/geschichte_umweltministerium.htm. Zugegriffen: 24. Febr. 2020.

Bhattacharya, Caroline. 2020. Gatekeeping the plenary floor: discourse network analysis as a novel approach to party control. Politics and Governance 8(2):229-242. https://doi.org/10.17645/pag.v8i2. 2611.

Brandes, Ulrik. 2008. On variants of shortest-path betweenness centrality and their generic computation. Social Networks 30(2):136-145. https://doi.org/10.1016/j.socnet.2007.11.001.

Brandes, Ulrik, und Dorothea Wagner. 2019. visone

Brandes, Ulrik, Patrick Kenis, Jörg Raab, Volker Schneider, und Dorothea Wagner. 1999. Explorations into the visualization of policy networks. Journal of Theoretical Politics 11(1):75-106. https://doi.org/10. $1177 / 0951692899011001004$.

Budge, Ian, und Dennis J. Farlie. 1983. Explaining and predicting elections: issue effects and party strategies in twenty-three democracies. London: George Allen \& Unwin Ltd.

Bundeswahlleiter. 2019. Ergebnisse Europawahl 2019. https://www.bundeswahlleiter.de/europawahlen/ 2019/ergebnisse/bund-99.html. Zugegriffen: 27. Febr. 2020.

Clauß, Anna. 2019. Bienenkönig Markus der Erste. https://www.spiegel.de/politik/bienenkoenig-markusder-erste-a-00000000-0002-0001-0000-000167197980. Zugegriffen: 12. Febr. 2020.

Dalton, Russell J. 1996. Citizen politics: Public opinion and political parties in advanced industrial democracies. Chatham: Chatham House.

Dalton, Russell J., und Martin P. Wattenberg. 2000. Parties without partisans: political change in advanced industrial democracies. Oxford: Oxford University Press.

Egleder, Julia. 2010. Umweltpolitik aus Verantwortung für die Schöpfung? CSU und Ökologie zwischen Programmatik, Publicity und Praxis. In Die CSU: Strukturwandel, Modernisierung und Herausforderungen einer Volkspartei, Hrsg. Gerhard Hopp, Martin Sebaldt, und Benjamin Zeitler, 207-218. Wiesbaden: VS.

Forschungsgruppe Wahlen. 2020. Politik II: Wichtige Probleme in Deutschland. https://www.forschungs gruppe.de/Umfragen/Politbarometer/Langzeitentwicklung_-_Themen_im_Ueberblick/Politik_II/ \#Probl1. Zugegriffen: 03. Aug. 2020. 
Glück, Alois. 2006. Bayern als Vorreiter in der Umweltpolitik. In Die Umweltmacher: 20 Jahre BMU Geschichte und Zukunft der Umweltpolitik, 109-120. Hamburg: Hoffmann und Campe.

Green-Pedersen, Christoffer. 2007. The growing importance of issue competition: the changing nature of party competition in western Europe. Political Studies 55(3):607-628. https://doi.org/10.1111/j. 1467-9248.2007.00686.x.

Green-Pedersen, Christoffer. 2019. Up and down with the environment. In The reshaping of west European party politics, 114-134. Oxford: Oxford University Press.

Green-Pedersen, Christoffer, und Peter B. Mortensen. 2010. Who sets the agenda and who responds to it in the Danish parliament? A new model of issue competition and agenda-setting. European Journal of Political Research 49(2):257-281. https://doi.org/10.1111/j.1475-6765.2009.01897.x.

Green-Pedersen, Christoffer, und Peter B. Mortensen. 2015. Avoidance and engagement: issue competition in multiparty systems. Political Studies 63(4):747-764. https://doi.org/10.1111/1467-9248.12121.

Guinaudeau, Isabelle, und Simon Persico. 2014. What is issue competition? Conflict, consensus and issue ownership in party competition. Journal of Elections, Public Opinion and Parties 24(3):312-333. https://doi.org/10.1080/17457289.2013.858344.

Heclo, Hugh. 1978. Issue networks and the executive establishment. In The new American political system, Hrsg. Anthony King, 78-124. Washington D.C.: American Enterprise Institute for Public Policy Research.

Holian, David B. 2004. He's stealing my issues! Clinton's crime rhetoric and the dynamics of issue ownership. Political Behavior 26(2):95-124. https://doi.org/10.1023/B:POBE.0000035959.35567.16.

Ingenhoff, Diana, und Ulrike Röttger. 2008. Issues Management. In Unternehmenskommunikation: Kommunikationsmanagement aus Sicht der Unternehmensführung, 2. Aufl., Hrsg. Miriam Meckel, Beat Schmid, 323-354. Wiesbaden: Gabler.

Jänicke, Martin. 2006. Umweltpolitik - auf dem Wege zur Querschnittspolitik. In Regieren in der Bundesrepublik Deutschland, Hrsg. Reimut Zohlnhöfer, Manfred G. Schmidt, 405-418. Wiesbaden: VS.

Kohout, Franz. 2013. Umweltpolitik. In Politik und Regieren in Bayern, Hrsg. Manuela Glaab, Michael Weigl, 331-341. Wiesbaden: Springer.

Lefevere, Jonas, Anke Tresch, und Stefaan Walgrave. 2015. Introduction: issue ownership. West European Politics 38(4):755-760. https://doi.org/10.1080/01402382.2015.1039375.

Leifeld, Philip. 2012. Discourse networks and German pension politics. Dissertation. Konstanz: Universität Konstanz.

Leifeld, Philip. 2016. Discourse network analysis: policy debates as dynamic networks. In The oxford handbook of political networks, Hrsg. Jennifer Alexander H.Montgomery Nicoll Victor, Mark Lubell, und Philip Leifeld, 1-28. Oxford: Oxford University Press.

Leifeld, Philip. 2019. Discourse network analyzer

Liermann, Sandra. 2019. Umfrage: Markus Söder ist beliebt wie lange nicht mehr: Bayern-Monitor. https:// www.augsburger-allgemeine.de/special/bayern-monitor/Umfrage-Markus-Soeder-ist-beliebt-wielange-nicht-mehr-id55554666.html. Zugegriffen: 10. Febr. 2020.

Lütz, Susanne. 2007. Policy-transfer und policy-diffusion. In Handbuch governance, Hrsg. Arthur Benz, Susanne Lütz, Uwe Schimank, und Georg Simonis, 132-143. Wiesbaden: VS.

Maier, Daniel, Annie Waldherr, Peter Miltner, Patrick Jähnichen, und Barbara Pfetsch. 2018. Exploring issues in a networked public sphere. Social Science Computer Review 36(1):3-20. https://doi.org/10. $1177 / 0894439317690337$.

Meguid, Bonnie. 2005. Competition between unequals: the role of mainstream party strategy in niche party success. American Political Science Review 99(3):347-359. https://doi.org/10.1017/ S0003055405051701.

Meguid, Bonnie. 2008. Party competition between unequals: strategies and electoral fortunes in Western Europe. Cambridge: Cambridge University Press.

Meyer, Thomas M., Martin Haselmayer, und Markus Wagner. 2017. Who gets into the papers? Party campaign messages and the media. British Journal of Political Science 50(1):281-302. https://doi.org/10. 1017/S0007123417000400.

Nagel, Melanie. 2016. Polarisierung im politischen Diskurs. Wiesbaden: Springer VS.

Neff, Benedict. 2018. Die CSU und die wankende Herrschaft im Bierzelt. https://www.nzz.ch/international/ wahl-in-bayern-csu-und-die-wankende-herrschaft-im-bierzelt-ld.1417935. Zugegriffen: 15. Jan. 2020.

Newman, Mark. 2010. Networks: an introduction. Oxford: Oxford University Press.

Petrocik, John. 1996. Issue ownership in presidential elections, with a 1980s case study. American Journal of Political Science 40(3):825-850. https://doi.org/10.2307/2111797. 
Polk, Jonathan, Jan Rovny, Ryan Bakker, Erica Edwards, Liesbet Hooghe, Seth Jolly, Jelle Koedam, Filip Kostelka, Gary Marks, Gijs Schumacher, Marco Steenbergen, Milada Vachudova, und Marko Zilovic. 2017. Explaining the salience of anti-elitism and reducing political corruption for political parties in Europe with the 2014 Chapel Hill Expert Survey data. Research \& Politics https://doi.org/10.1177/ 2053168016686915.

Riker, William H. 1996. The strategy of rhetoric: campaigning for the American constitution. New Haven, London: Yale University Press.

Robertson, David. 1976. A theory of party competition. London, New York: Wiley.

Sabatier, Paul A., und Christoph M. Weible. 2007. The advocacy coalition framework: innovations and clarifications. In Theories of the policy process, Hrsg. Paul A. Sabatier, 189-220. London: Routledge.

Schneider, Volker. 2015. Hugh Heclo, “issue networks and the executive establishment". In The Oxford handbook of classics in public policy and administration, Hrsg. Martin Edward C.Page Lodge, Steven J. Balla, und Volker Schneider. Oxford: Oxford University Press.

Schneider, Volker, Frank Janning, Philip Leifeld, und Thomas Malang (Hrsg.). 2009. Politiknetzwerke. Wiesbaden: VS.

Seeberg Bech, Henrik. 2020. First avoidance, then engagement: political parties' issue competition in the electoral cycle. Party Politics https://doi.org/10.1177/1354068820970353.

Smith, Tom W. 1985. The polls: America's most important problems part I: national and international. The Public Opinion Quarterly 49(2):264-274. https://doi.org/10.1086/268922.

Spoon, Jae-Jae, Sarah B. Hobolt, und Catherine E. de Vries. 2014. Going green: explaining issue competition on the environment. European Journal of Political Research 53(2):363-380. https://doi.org/10. 1111/1475-6765.12032.

Tratzmiller, Johannes Philipp. 2018. Die Kehrtwende in der deutschen Atompolitik nach Fukushima: Detailanalyse eines politischen Entscheidungsprozesses. Dissertation. Konstanz: Universität Konstanz. 